\title{
ON PERTURBATIONS OF IDEAL COMPLEMENTS
}

\author{
BORIS SHEKHTMAN
}

To Nigel Kalton on occasion of his 60th birthday.

\begin{abstract}
Let $\mathbb{F}[x]$ be the space of polynomials in $d$ variables, let $\mathfrak{G}^{N}$ be the Grassmannian of $N$-dimensional subspaces of $\mathbb{F}[x]$ and let $J_{N}$ stand for the family of all ideals in $\mathbb{F}[x]$ of codimension $N$. For a given $G \in \mathfrak{G}^{N}$ we let

$$
\mathfrak{J}_{G}:=\left\{J \in \mathfrak{J}_{N}: J \cap G=\{0\}\right\}
$$

Is it true, that (with appropriate topology on $\mathfrak{J}_{N}$ ) the set $\mathfrak{J}_{G}$ is dense in $\mathfrak{J}_{N}$ ? In general the answer is "No". What is even more surprising, that there are "good ideals" $J \in \mathfrak{J}_{N}$ such that every "neighborhood" $\mathcal{U}(J) \subset \mathfrak{J}_{N}$ has a non-empty intersection with $\mathfrak{J}_{G}$ for any $G \in \mathfrak{G}^{N}$ and there are "bad" ideals $J \in \mathfrak{J}_{N}$ (for $d \geq 3$ ) such that some "neighborhoods" $\mathcal{U}(J) \subset \mathfrak{J}_{N}$ have an empty intersection with $\mathfrak{J}_{G}$ for some $G \in \mathfrak{G}^{N}$. This contrast illuminates the non-homogeneous nature of $\mathfrak{J}_{N}$.
\end{abstract}

\section{Preliminaries}

Let $F$ be a normed linear space, let $\mathfrak{G}^{N}(F)$ be the Grassmannian of $N$-dimensional subspaces of $F$ and let $\mathfrak{G}_{N}(F)$ denotes the Grassmannian of all subspaces of $F$ of codimension $N$. For a given $G \in \mathfrak{G}_{N}(F)$ let

$$
\mathfrak{G}_{G}(F):=\left\{J \in \mathfrak{G}_{N}(F): J \cap G=\{0\}\right\}
$$

i.e., $\mathfrak{G}_{G}(F)$ is a family of all subspaces $J$ in $F$ that are "missing" $G$ or, equivalently, the family of all subspaces of $F$ that complement $G$.

It is well-known and easy to see that (with appropriate topology on $\mathfrak{G}_{N}(F)$ ) for any $G \in \mathfrak{G}^{N}(F)$, the set $\mathfrak{G}_{G}(F)$ is an open and dense subset of $\mathfrak{G}_{N}(F)$.

The main focus of this article is an investigation of the following "ideal" version of this statement.

Let $F=\mathbf{F}[x]=\left[x_{1}, \ldots, x_{d}\right]$ be the space of polynomials in $d$ variables over the field $\mathbb{F}$ of real or complex numbers. Let $J_{N}$ stand for the family of all ideals in $\mathbb{F}[x]$ of codimension $N$. Let $\mathfrak{G}^{N}:=\mathfrak{G}^{N}(\mathbb{F}[x])$. For a given $G \in \mathfrak{G}^{N}$ we let

$$
\mathfrak{J}_{G}:=\left\{J \in \mathfrak{J}_{N}: J \cap G=\{0\}\right\}
$$

Question : Is it still true, that $\mathfrak{J}_{G}$ is dense in $\mathfrak{J}_{N}$ ?

In general the answer is "No". What is even more surprising, that there are "good ideals" $J \in \mathfrak{J}_{N}$ such that every "neighborhood" $\mathcal{U}(J) \subset \mathfrak{J}_{N}$ has a non-empty intersection with $\mathfrak{J}_{G}$ for any $G \in \mathfrak{G}^{N}$ and there are "bad" ideals $J \in \mathfrak{J}_{N}$ (for $d \geq 3$ ) such that some "neighborhoods" $\mathcal{U}(J) \subset \mathfrak{J}_{N}$ have an empty intersection with $\mathfrak{J}_{G}$ for some $G \in \mathfrak{G}^{N}$. This contrast illuminates the non-homogeneous nature of $\mathfrak{J}$ (as oppose to $\mathfrak{G}_{N}$ ).

1991 Mathematics Subject Classification. Primary 46H10,46J05,46J20; Secondary 14 C05.

Key words and phrases. Ideal complements, Ideal projections. 
We will use the rest of this section to describe the topology on $\mathfrak{J}_{N}$. Actually, any norm on $\mathbb{F}[\mathbf{x}]$ and any "reasonable" notion of convergence of a sequences of ideals in $\mathfrak{J}_{N}$ will lead to the same results. The underlined reason for it is the existence of intrinsic (Zariski) topology on the Hilbert scheme $\mathfrak{F}^{N}\left(\mathbb{F}^{d}\right)$ parametrizing $\mathfrak{J}_{N}$, which is formally weaker then any reasonable topology (cf. [6]).

For the sake of specificity, define the norm of $f=\sum \hat{f}(\mathbf{k}) \mathbf{x}^{\mathbf{k}} \in \mathbb{F}[\mathbf{x}]$ by

$$
\|f\|:=\sum_{\mathbf{k}}|\hat{f}(\mathbf{k})|,
$$

which turns $\mathbb{F}[\mathbf{x}]$ into a normed space with continuous multiplication. Let $(\mathbb{F}[\mathbf{x}])^{\prime}$ be the dual of $\mathbb{F}[\mathbf{x}]$. An ideal $J \in \mathfrak{J}_{N}$ induces an $N$-dimensional subspace $J^{\perp} \subset$ $(\mathbb{F}[\mathbf{x}])^{\prime}$ defined as

$$
J^{\perp}:=\left\{\lambda \in(\mathbb{F}[\mathbf{x}])^{\prime}: \lambda(f)=0, \forall f \in J\right\}
$$

that uniquely identifies the ideal $J$ via $\left(J^{\perp}\right)^{\top}=J$. We will adopt the following definition of convergence:

Definition 1.1. Let $\left(J_{m}, m \in \mathbb{N}\right)$ be a sequence of ideals in $\mathfrak{J}_{N}$ and let $J \in \mathfrak{J}_{N}$. We say that $J_{m} \rightarrow J$ if for every $\lambda \in J^{\perp}$ there exists $\lambda_{m} \in J_{m}^{\perp}$ such that

$$
\lambda_{m}(f) \longrightarrow \lambda(f)
$$

for every $f \in \mathbb{F}[\mathbf{x}]$.

A simple perturbation argument yields the following:

Theorem 1.2. (cf $[8])$ Let $\left(J, J_{m}, m \in \mathbb{N}\right)$ be a sequence of ideals in $\mathfrak{J}_{N}$ such that $J_{m} \rightarrow J$. Let $E \in \mathfrak{G}^{N}$ complements $J$. Then the space $E$ complements $J_{m}$ for sufficiently large $m$ and

$$
P_{m} f \rightarrow P f
$$

for all $f \in \mathbb{F}[\mathbf{x}]$, where $P_{m}$ and $P$ are projections onto $E$ with ker $P_{m}=J_{m}$, ker $P=J$.

Conversely, let $P_{m}$ and $P$ be projections onto a space $E$ with ideal kernels, such that (1.2) holds. Then $\operatorname{ker} P_{m} \rightarrow \operatorname{ker} P$.

Definition 1.3. (Birkhoff, [1]). A linear idempotent operator $P$ on $\mathbb{F}[\mathbf{x}]$ is called an ideal projection if $\operatorname{ker} P$ is an ideal in $\mathbb{F}[\mathbf{x}]$.

The symbol $\mathfrak{P}_{N}$ will stand for all $N$-dimensional ideal projections and for a $G \in \mathfrak{G}^{N}$ we let $\mathfrak{P}_{G}$ be the family of all ideal projections onto $G$. Thus $\mathfrak{J}_{G}$ is in one-to-one correspondence with $\mathfrak{P}_{G}$.

A nice characterization of ideal projections is due to C. de Boor [2]:

Proposition 1.4. Let $P$ be a linear mapping on $\mathbb{F}[\mathbf{x}]$. Then $P$ is an ideal projection if and only if

$$
P(f g)=P(f \cdot P g)
$$

for all $f, g \in \mathbb{F}[\mathbf{x}]$.

In one variable, the space $F_{<N}[x]$ of polynomials of degree less than $N$ complements every ideal $J \in \mathfrak{J}_{N}$ (cf. proof of Proposition 2.1 below). In two or more variables there does not exist a subspace $G \in \mathfrak{G}^{N}$ with this property. However: 
Theorem 1.5. For every $N$ and $d$ there exists a fixed finite family $\mathcal{E}^{N}$ of $N$ dimensional (translation-invariant and spanned by monomials) subspaces of $\mathbb{F}[\mathbf{x}]$ such that every $J \in \mathfrak{J}_{N}$ complements at least one $E \in \mathcal{E}^{N}$.

Let $\mathbb{F}_{<N}[\mathbf{x}]$ be the space of polynomials of degree less than $N$ and $\mathbb{F}_{\leq N}[\mathbf{x}]$ be the space of polynomials of degree at most $N$. It follows from Theorem 1.5 that every $E \in \mathcal{E}^{N}$ is a subspace of $\mathbb{F}_{<N}[\mathbf{x}]$, i.e., $\mathcal{E}^{N} \subset \mathfrak{G}^{N}\left(\mathbb{F}_{<N}[\mathbf{x}]\right)$

The next theorem is a consequence of Theorem 1.5 and the de Boor's formula.

Theorem 1.6. Let $\left(J, J_{m}, m \in \mathbb{N}\right)$ be a sequence of ideals in $\mathfrak{J}_{N}$. Then $J_{m} \rightarrow J$ if and only if there exists a subspace $E \in \mathbb{F}_{<N}[\mathbf{x}]$ and a sequence of ideal projections $\left(P, P_{m}, m>M\right)$ such ker $P=J, \operatorname{ker} P_{m}=J_{m}$ and

$$
\left\|P-P_{m}\right\|_{\mathbb{F} \leq N[\mathbf{x}]} \rightarrow 0 .
$$

Proof. If $J \in \mathfrak{J}_{N}$ then by Theorem 1.5 there exists a subspace $E \subset \mathbb{F}_{<N}[\mathbf{x}]$ that complements $J$ and since $\mathbb{F}_{\leq N}[\mathbf{x}]$ is finite-dimensional, by the Theorem 1.2, (1.3) implies (1.4).

Conversely, suppose that $f \in \mathbb{F}_{\leq K}[\mathbf{x}]$ for some $K \in \mathbb{N}$. If $K \leq N$ then (1.2) follows from (1.4). Assuming (1.2) for all monomials $f \in \mathbb{F}_{\leq K}[\mathbf{x}]$, let $g$ be a monomial of degree $K+1$. Then $g=x_{j} f$ for some $j=1, \ldots, d$ and by (1.3):

$$
\left\|P g-P_{m} g\right\|_{\mathbb{F}_{\leq N}[\mathbf{x}]}=\left\|P\left(x_{j} P f\right)-P_{m}\left(x_{j} P_{m} f\right)\right\|_{\mathbb{F}_{\leq N}[\mathbf{x}]} \rightarrow 0
$$

since $x_{j} P f, x_{j} P_{m} f \in \mathbb{F}_{\leq N}[\mathbf{x}]$ and $x_{j} P_{m} f \rightarrow x_{j} P f$ by inductive assumption. Hence (1.2) holds for all monomials.

This theorem allows us to define an $\varepsilon$-neighborhood of an ideal $J \in \mathfrak{J}_{N}$ : Let $E \in \mathcal{E}^{N}$ be such that $J \in \mathfrak{J}_{E}$. Let $P$ be an ideal projection onto $E$ with $\operatorname{ker} P=E$. Define

$$
\mathcal{U}(E, J, \varepsilon)=\left\{\operatorname{ker} Q,: Q \in \mathfrak{P}_{E},\|P-Q\|_{\mathbb{F}_{\leq N}[\mathbf{x}]}<\varepsilon\right.
$$

and

$$
\mathcal{U}(J, \varepsilon)=\underset{J \in \mathfrak{J}_{E}}{\cap} U(E, J, \varepsilon)
$$

\section{2. "Good IdEALS"}

We will now examine closely one special space $E \in \mathcal{E}^{N}$ :

$$
E:=\operatorname{span}\left\{1, x_{1}, x_{1}^{2}, \ldots, x_{1}^{N-1}\right\}
$$

viewed as an $N$-dimensional subspace of $\mathbb{F}[\mathbf{x}]=\mathbb{F}\left[x_{1}, x_{2}, \ldots, x_{d}\right]$. Let $P$ be an ideal projection onto $E$. Then

$$
\begin{gathered}
P\left(x_{1}^{N}\right)=p_{1}=\sum_{j=0}^{N-1} b_{1, j} x_{1}^{j}, \\
P\left(x_{2}\right)=p_{2}=\sum_{j=0}^{N-1} b_{2, j} x_{1}^{j}, \\
\vdots \\
P\left(x_{d}\right)=p_{d}=\sum_{j=0}^{N-1} b_{d, j} x_{1}^{j} .
\end{gathered}
$$

Let $B$ be the collection of coefficients

$$
B=\left(b_{k, j}, k=1, \ldots, d ; j=0, \ldots, N-1\right)
$$


Then, by the de Boor's formula (1.3), we have

$$
\begin{aligned}
P\left(x_{1}^{N+1}\right) & =P\left(x_{1} P x_{1}^{N}\right)=P\left(x_{1}\left(\sum_{j=0}^{N-1} b_{1, j} x_{1}^{j}\right)\right) \\
& =P\left(\sum_{j=0}^{N-1} b_{1, j} x_{1}^{j+1}\right)=b_{1, N-1}\left(\sum_{j=0}^{N-1} b_{1, j} x_{1}^{j}+\sum_{j=0}^{N-2} b_{1, j} x_{1}^{j+1}\right) \\
& =b_{1, N-1} b_{1,0}+\sum_{j=1}^{N-1} b_{1, N-1}\left(b_{1, j}+b_{1, j-1}\right) x_{1}^{j} .
\end{aligned}
$$

Inductively, we conclude that

$$
P\left(x_{1}^{k}\right)=\sum_{j=0}^{N-1} q_{k, j}(B) x_{1}^{j}
$$

for all $k$, where $q_{k, j} \in \mathbb{F}[B]$ are polynomials in $d N$ variables $B$. Now, using $P\left(x_{j} f\right)=$ $P\left(f P x_{j}\right)$ for every $f \in E$ we conclude that

$$
P f=\sum_{j=0}^{N-1} q_{f, j}(B) x_{1}^{j}
$$

for $f \in x_{j} E$, where $q_{f, j} \in \mathbb{F}[B]$. Inductively, (1.8) holds for all $f \in \mathbf{x}^{\mathbf{k}} E$ and therefore for all $f \in \mathbb{F}[\mathbf{x}]$ with $q_{f, j} \in \mathbb{F}[B]$.

Hence a sequence of $d$ polynomials $\left(p_{1}, \ldots, p_{d}\right)$ given by (1.4) (or equivalently the sequence of $d N$ coefficients $B$ ) completely determines the ideal projector $P$ onto $E$. What so special about this particular space $E$ is that the converse also holds:

Proposition 2.1. Every sequence $\left(p_{1}, \ldots, p_{d}\right)$ of polynomials in $E$ defines an ideal $J=\left\langle x_{1}^{N}-p_{1}, x_{2}-p_{2}, \ldots, x_{d}-p_{d}\right\rangle$ that complements $E$. Hence every sequence $B$ of $d N$ scalars defines an ideal projection $P_{B}$ onto $E$ by (2.4) and every ideal projection $P$ onto $E$ defines a sequence $B_{P}$ by (2.2). Clearly

$$
P_{B_{P}}=P \text { and } B_{P_{B}}=B \text {. }
$$

Proof. It is clear from the construction of $E$ that $E \cap J=\{0\}$. Let $f \in \mathbb{F}\left[x_{1}\right]$ be a polynomial in only one variable. Using the division algorithm in $\mathbb{F}\left[x_{1}\right]$ we have $f=q\left(x_{1}^{N}-p_{1}\right)+r$ with $\operatorname{deg} r<N$. Thus the ideal $\left\langle x_{1}^{N}-p_{1}\right\rangle$ generated by $x_{1}^{N}-p_{1}$ complements the space $\mathbb{F}_{<N}\left[x_{1}\right]$ of polynomials of degree less than $N$ in $\mathbb{F}\left[x_{1}\right]$ and $E+J \supset \mathbb{F}\left[x_{1}\right]$. Inductively, we assume that $E+J \supset \mathbb{F}\left[x_{1}, \ldots, x_{k}\right], k<d$ and prove that $E+J \supset \mathbb{F}\left[x_{1}, \ldots, x_{k}, x_{k+1}\right]$, i.e., we need to show that $x_{k+1}^{n} \mathbb{F}\left[x_{1}, \ldots, x_{k}\right] \subset E+J$ for all $n$. For $f \in \mathbb{F}\left[x_{1}, \ldots, x_{k}\right]$ we have

$$
x_{k+1} f=\left(x_{k+1}-p_{k+1}\right) f+p_{k+1} f \in E+J
$$

since the first term is in the ideal $J$ and the second belongs to $E+J$ by inductive assumption. Using induction on $n$, assume that $f \in x_{k+1}^{n} \mathbb{F}\left[x_{1}, \ldots, x_{k}\right]$ and conclude that $x_{k+1} f \in x_{k+1}^{n+1} \mathbb{F}\left[x_{1}, \ldots, x_{k}\right]$ has a representation (2.5).

Corollary 2.2. $B_{m} \rightarrow B$ if and only if $\operatorname{ker} P_{B_{m}} \rightarrow \operatorname{ker} P_{B}$.

Proof. Since $q_{f, j}(B)$ in (2.4) are polynomials (hence continuous function of $B$ ) $B_{m} \rightarrow B$ implies $P_{B_{m}} f \rightarrow P_{B} f$ for every $f$. Conversely, if $P_{B_{m}} f \rightarrow P_{B} f$ then 
$P_{B_{m}} x_{1}^{N} \rightarrow P_{B} x_{1}^{N}$ and $P_{B_{m}} x_{j} \rightarrow P_{B} x_{j}$ for all $j=2, \ldots, d$. Now $B_{m} \rightarrow B$ follows from (2.2).

We are now ready to prove that every ideal $J \in \mathfrak{J}_{E}$ is a "good ideal".

Theorem 2.3. Let $J \in \mathfrak{J}_{E}$ and $G \in \mathfrak{G}^{N}$. Then every neighborhood $\mathcal{U}(J)$ has a non-empty intersection with $\mathfrak{J}_{G}$.

Proof. First, we establish that $\mathfrak{J}_{E} \cap \mathfrak{J}_{G} \neq \varnothing$. Let $g_{1}, \ldots, g_{N}$ be a bases in $G$ and $e_{1}, \ldots, e_{N}$ be a bases in $E$. There exists a sequence $\left\{\mathbf{z}_{1}^{*}, \ldots, \mathbf{z}_{N}^{*}\right\}$ of points in $\mathbb{F}^{d}$ such that $g_{j}\left(\mathbf{z}_{k}^{*}\right)=\delta_{j, k}$ and hence the polynomial

$$
\operatorname{det}\left(g_{j}\left(\mathbf{z}_{k}\right), j=1, \ldots N\right)
$$

in $d N$ variables (coordinates of the points $\mathbf{z}_{k}$ ) is not identically zero. Therefore the set

$$
\mathcal{Z}_{G}:=\left\{\left(\mathbf{z}_{1}, \ldots, \mathbf{z}_{N}\right): \operatorname{det}\left(g_{j}\left(\mathbf{z}_{k}\right)\right) \neq 0\right.
$$

is an open and dense set in $\left(\mathbb{F}^{d}\right)^{N}$. Similarly the set

$$
\mathcal{Z}_{E}:=\left\{\left(\mathbf{z}_{1}, \ldots, \mathbf{z}_{N}\right): \operatorname{det}\left(e_{j}\left(\mathbf{z}_{k}\right)\right) \neq 0\right.
$$

is an open and dense set in $\left(\mathbb{F}^{d}\right)^{N}$. Thus $\mathcal{Z}_{G} \cap \mathcal{Z}_{E} \neq \varnothing$ and for any $\left(\mathbf{z}_{1}, \ldots, \mathbf{z}_{N}\right) \in$ $\mathcal{Z}_{G} \cap \mathcal{Z}_{E}$, the ideal

$$
J:=\left\{f \in \mathbb{F}[\mathbf{x}]: f\left(\mathbf{z}_{j}\right)=0, j=1, \ldots N\right\}
$$

complements $E$ and $G$.

Therefore, there exists a sequence $B^{*} \in \mathbb{F}^{d N}$ such that $P_{B^{*}}$ is an ideal projection onto $E$ and ker $P_{B^{*}} \in \mathfrak{J}_{G}$. Let

$$
\mathcal{B}_{G}:=\left\{B \in \mathbb{F}^{d N}: \operatorname{ker} P_{B} \in \mathfrak{J}_{G}\right\} .
$$

It follows that $\mathcal{B}_{G} \neq \varnothing$. Suppose that $\operatorname{ker} P_{B} \in \mathfrak{J}_{G}$ i.e., $\operatorname{ker} P_{B} \cap G=\{0\}$. Then

$$
P_{B}\left(\sum_{k=1}^{N} \alpha_{k} g_{k}\right)=\sum_{k=1}^{N} \alpha_{k} P_{B} g_{k}=0
$$

implies $\alpha_{k}=0$ for all $k=1, \ldots, N$. Hence $\operatorname{ker} P_{B} \in \mathfrak{J}_{G}$ is the same as linear independency of the sequence of polynomials $\left(P_{B} g_{k}, k=1, \ldots, N\right)$. Since, by $(2.4)$, $P_{B} f=\sum_{j=0}^{N-1} q_{g_{k}, j}(B) x_{1}^{j}$ this is equivalent to

$$
\operatorname{det}\left(q_{g_{k}, j}(B)\right) \neq 0 \text {. }
$$

Since $\mathcal{B}_{G} \neq \varnothing$, this determinant is a non-zero polynomial in $\mathbb{F}[B]$, hence there exists an open and dense set of $B \subset \mathbb{F}^{d N}$ such that $\operatorname{ker} P_{B} \in \mathfrak{J}_{G}$.

\section{3. "BAD IDEALS"}

In this section we will use a beautiful construction of A. Iarrobino and modified the reasoning of [7] to show that for $d \geq 3$ and for sufficiently large $N$ there exists an ideal $J \in \mathfrak{J}_{N}$ such that $J$ is not the limit of ideals in $\mathfrak{J}_{E}$, where $E:=\operatorname{span}\left\{1, x_{1}, x_{1}^{2}, \ldots, x_{1}^{N-1}\right\}$ is the space considered in the previous section.

Let $W:=M_{<n}^{d}[\mathbf{x}]$ be the set of monomials of degree less than $n$. Let $U \cup V=$ $M_{n}^{d}[\mathbf{x}]$ be a non-trivial partition of the set $M_{n}^{d}[\mathbf{x}]$ of all monomials of degree $n$ in $\mathbb{F}[\mathbf{x}]$. Let $H$ be the subspace of $\mathbb{F}[\mathbf{x}]$ spanned by $\mathbb{F}_{<n}^{d}[\mathbf{x}]$ and $V$ and let

$$
N=\operatorname{dim} H=\# V+\# W
$$


For any choice of matrix $C \in \mathbb{F}^{U \times V}$, the space $J_{C}$ spanned by monomials of degree greater than $n$ and the specific polynomials

$$
p_{u}:=u-\sum_{v \in V} C(u, v) v, u \in U,
$$

complements $H$ and is an ideal. The latter is so because each $p_{u}$ is homogeneous, thus every product of a monomial with $p_{u}$ is in $J_{C}$. Furthermore, as is easy to see

$$
\mathbb{F}[\mathbf{x}]=H \oplus J_{C} .
$$

for each $C$.

Theorem 3.1. For any $d \geq 3$ and for sufficiently large $n$, there exists a partition $U \cup V=M_{n}^{d}[\mathbf{x}]$ and a matrix $C \in \mathbb{F}^{U \times V}$ such that the ideal $J_{C}$ can not be perturbed to complement $E$.

Proof. Assume that $J_{C}$ complements $H$ and that there is a small perturbation of $J_{C}$ that complements $E$. In other words, assume the existence of a sequence of ideals $\left\{J_{m}\right\}$ such that each $J_{m}$ complements $G$ and $J_{m} \rightarrow J_{C}$. This is the same as the existence of a sequence of ideal projections $P_{m}$ onto $H$ such that $\operatorname{ker} P_{n}$ complements $E$ and $H$ at the same time and $P_{m} f \rightarrow P f$ for every $f \in \mathbb{F}^{d}[\mathbf{x}]$. In particular

$$
u-P_{m} u \rightarrow u-\sum_{v \in V} C(u, v) v, \forall u \in U
$$

Since $P_{m}$ is a projection onto $H$ it follows that

$$
P_{m} u=\sum_{v \in V} C_{m}(u, v) v+\sum_{w \in W} C_{m}(u, w) w
$$

and

$$
C_{m}(u, v) \rightarrow C(u, v), \forall u \in U \text { and } C_{m}(u, w) \rightarrow 0, \forall w \in W .
$$

Since ker $P_{m}$ complements $E$, we let $Q_{m}$ be the ideal projection onto $E$ with $\operatorname{ker} Q_{m}=\operatorname{ker} P_{m}$. Then $u-P_{m} u \in \operatorname{ker} P_{m}=\operatorname{ker} Q_{m}$ and

$$
0=Q_{m}\left(u-P_{m} u\right)=Q_{m} u-\left(\sum_{v \in V} C_{m}(u, v) Q_{m} v+\sum_{w \in W} C_{m}(u, w) Q_{m} w\right)
$$

or

$$
\sum_{v \in V} C_{m}(u, v) Q_{m} v+\sum_{w \in W} C_{m}(u, w) Q_{m} w=Q_{m} u
$$

At this point it is important to notice that, as projections onto $E$, the operators $Q_{m}$ depend polynomially on $d \times N$ parameters $B$ as in $(2,4)$ :

$$
Q_{m} f=\sum_{k=0}^{N-1} q_{k, f}^{(m)}(B) x_{1}^{k}
$$

where $q_{k, f}^{(n)} \in \mathbb{F}^{d N}[B]$. Rewriting (3.6) we have:

$$
\sum_{k=0}^{N-1}\left(\sum_{v \in V} C_{m}(u, v) q_{k, v}^{(m)}(B)+\sum_{w \in W} C_{m}(u, W) q_{k, w}^{(m)}(B)\right) x_{1}^{k}=\sum_{k=0}^{N-1} q_{k, u}^{(m)}(B) x_{1}^{k}
$$


or equivalently

$$
\sum_{v \in V}^{(3.9)} C_{m}(u, v) q_{k, v}^{(m)}(B)+\sum_{w \in W} C_{m}(u, w) q_{k, w}^{(m)}(B)=q_{k, u}^{(m)}(B), u \in U, k=0, \ldots, N-1 .
$$

Since $\# V+\# W=N$, this is the system of $N \times \# U$ equations with the same number of unknowns $\left\{C_{m}(u, v), u \in U\right\}$ and $\left\{C_{m}(u, w), u \in U\right\}$. By Cramer's rule

$$
C_{m}(u, v)=\frac{\operatorname{det} V_{m, u, v}(B)}{\operatorname{det} V_{m}(B)},
$$

where $\operatorname{det} V_{m}(B)$ is the determinant of the matrix on the left-hand side of (3.9), and $\operatorname{det} V_{m, u, v}(A)$ is the determinant of the same matrix with the $(u, k)$-th column replaced by the column $\left(q_{k, u}^{(m)}(B)\right)$. Notice that that makes $C_{m}(u, v)$ rational function of $d \times N$ parameters $B$ with common denominator. Thinking of $1 / \operatorname{det} V_{m}(B)$ as just another variable, say $z$, we conclude that the set

$$
\mathfrak{C}:=\left\{z \operatorname{det} V_{n, u, v}(B), B \in \mathbb{F}^{d N}, z \in \mathbb{F} \backslash\{0\}\right\} \subset \mathbb{F}^{\# U \times \# V}
$$

is a polynomial image of $\mathbb{F}^{d N+1}$ where the first $d N$ parameters are the parameters of $B$. As such $\mathfrak{C}$ forms an affine subvariety of $\mathbb{F} \# U \times \# V$ with $\operatorname{dim} \mathfrak{C} \leq d N+1$ (cf [4], Theorem 2, p. 466). Hence if

$$
\# U \times \# V>d N+1
$$

then there exists a collection $C(u, v) \in \mathbb{F}^{\# U \times \# V}$ which is not in the closure of $\mathfrak{C}$, contradicting $(3,5)$.

Now we only need to count. As was pointed out in [5], we have $\# M_{n}^{d}[\mathbf{x}]=$ $\left(\begin{array}{c}n+d-1 \\ d-1\end{array}\right) \approx\left(\frac{n^{d-1}}{(d-1) !}\right)$, choosing the partition $U \cup V=M_{n}^{d}[\mathbf{x}]$ such that $\# U=$ $\left\lfloor\frac{1}{2}\left(\begin{array}{c}n+d-1 \\ d-1\end{array}\right)\right\rfloor$ we have

$$
\# U \times \# V=\left\lfloor\frac{1}{2}\left(\begin{array}{c}
n+d-1 \\
d-1
\end{array}\right)\right\rfloor\left\lceil\frac{1}{2}\left(\begin{array}{c}
n+d-1 \\
d-1
\end{array}\right)\right\rceil \approx \frac{1}{4}\left(\frac{n^{d-1}}{(d-1) !}\right)^{2} \approx n^{2 d-2}
$$

while $N=\operatorname{dim} H=\operatorname{dim} \mathbb{F}_{\leq n}^{d}[\mathbf{x}]-\# U=\left(\begin{array}{c}n+d \\ d\end{array}\right)-\left\lfloor\frac{1}{2}\left(\begin{array}{c}n+d-1 \\ d-1\end{array}\right)\right\rfloor \approx \frac{n^{d}}{d !} \approx n^{d}$. Hence for sufficiently large $n,(3.11)$ holds. Direct computation yield (3.11) with $n=7$, for $d=3 ; n=3$ for $d=4$ or 5 and $n=2$ for $d>5$.

Remark 3.2. The "bad" ideals do not exist in $\mathbb{F}[\mathbf{x}]$ for $d=1$, as follow from the Theorem2.3, since, for $d=1$, every ideal in $\mathfrak{J}_{N}$ is complemented to

$$
E:=\operatorname{span}\left\{1, x_{1}, x_{1}^{2}, \ldots, x_{1}^{N-1}\right\}
$$

"Bad" ideals also do not exist in $\mathbb{C}[\mathbf{x}]$ for $d=2$ as noted in [8]. The existence of "bad" ideals in $\mathbb{R}[\mathbf{x}]$ for $d=2$ is an open problem.

\section{REFERENCES}

[1] Birkhoff, G. The Algebra of Multivariate Interpolation, in "Constructive approaches to mathematical models." (C.V. Coffman and G.J.Fix Eds.), pp345-363, Academic Press, New-York, 1979

[2] de Boor, C. Ideal Interpolation, Approximation Theory XI: Gatlinburg 2004, C. K. Chui, M. Neamtu and L.L.Schumaker (eds.), Nashboro Press (2005), pp.59-91.

[3] Cox, D., J.Little and D. O'Shea, Using Algebraic Geometry, Graduate Texts in Mathematics, Springer-Verlag, New-York-Berlin-Heidelberg, 1997. 
[4] Cox, D., J.Little and D. O'Shea, Ideals,Varieties, and Algorithms, (second edition), SpringerVerlag, New-York-Berlin-Heidelberg, 1997.

[5] Iarrobino, A. Reducibility of the Families of 0-dimensional Schemes on a Variety, Inventiones Math. 15,

(1972) pp.72-77.

[6] Mumford, D., The Red Book of Varieties and Schemes, Lecture Notes in Mathematics 1358, Springer-Verlag 1988.

[7] Shekhtman, B., On a Conjecture of Carl de Boor Regarding the Limits of Lagrange Interpolants. Constr. Approx. 3, (2006) 24: 365-370

[8] Shekhtman, B., On Bivariate Ideal Projectors and their Perturbations, Advances in Comput. Math. (to appear).

Department of Mathematics,, University of South Florida,, Tampa, Fl. 33620

E-mail address: boris@math.usf.edu 